sei. Der Comet Ross hatte nach den Elementen von Dr. Oppenheim um diese Zeit die Position $18^{\mathrm{h}} 10^{\mathrm{m}}-1 \mathbf{1}^{\circ} \mathbf{2}^{\prime}$. Der Aufgang der Sonne findet an diesem Tage $2 \mathrm{a}$ New Norfolk um $16^{\mathrm{h}} 26^{\mathrm{m}}$ Ortszeit; der des Cometen erst um $1 \eta^{\mathrm{h}} \mathrm{o}^{\mathrm{m}}$ statt, so dass er, falls man den vorstehenden Elementen keine zu grosse Unsicherheit zuschreiben will, nicht sichtbar gewesen sein kann.

Ein weiterer Umstand, der dagegen spricht, dass die angefuhrte Notiz sich auf den Cometen Ross bezieht, ist der folgende. Herr Ellery erwäbnt in Nr. 84 des ,Observatory, dass der Comet am I I. Januar die Helligkeit eines Sternes 10. Grösse gehabt habe. Da nun am 26. Dec. der Comet nur das fünffache dieser Helligkeit besessen hat, so ist es schon aus diesem Grunde nicht anzunehmen, dass er in New Norfolk, falls er wirklich sichtbar gewesen wäre, eine auffallende Erscheinung dargeboten habe.

Kiel 1884 April 3 .

H. Kreutz.

\title{
Note on the proper motion of Lal. 13849.
}

This star is called a sixth magnitude in Weisse, and a seventh in Lalande; their places differ considerably in Declination. gives:

The position for 1884.0 reduced from Baily's Lalande

$$
7^{h} 3^{m} 14: 56+21^{\circ} 27^{\prime} 36^{\prime \prime} \cdot 0
$$

Bessel's place reduced from Weisse gives:

$$
7^{\mathrm{h}} 3^{\mathrm{m}} \times 4^{:} 3^{\circ}+21^{\circ} 27^{\prime} 19^{\prime \prime} 4
$$

The mean of two transit observations on Feb. $29^{\text {th }}$ and March $4^{\text {th }} 1884$ by. Mr. Paul at the Naval Observatory gives:

$$
7^{\mathrm{h}} 3^{\mathrm{m}} 13^{: 60}+21^{\circ} 26^{\prime} 49^{\circ: 8}
$$

The $1^{\text {tt }}$ and $2^{\text {nd }}$ observations were made by Lalande on March $2^{\text {nd }} 1797$ by Bessel on March I $1^{\text {th }} 1825$
From the $1^{\text {st }}$ and $3^{\text {rd }}$. of these places we obtain an annual proper motion of:

$$
-0.0110 \text { and }-0.531
$$

From the $2^{\text {nd }}$ and $3^{\text {rid }}$

$$
\text { -- 0.0117 and -0."502 }
$$

Correcting for proper motion from the last observations to the beginning of the year we obtain the position for 1884.0 :

$$
7^{\mathrm{h}} 3^{\mathrm{m}} 13^{3} \cdot 60+21^{\circ} 26^{\prime} 49^{\prime \prime} \cdot 9
$$

\begin{tabular}{|c|c|c|c|c|c|c|c|}
\hline I 884 & Melb. M. T. & & $\Delta \alpha$ & $\Delta \delta$ & $\alpha$ app. & $\delta$ app. & * \\
\hline Jan. 18 & $8^{h} 45^{m} 5^{6 s}$ & + & $2^{m} 11: 0$ & + I I' 20.9 & $23^{h} 32^{m} 29^{s}: 8$ & $-7^{\circ} 33^{\prime} 5^{8^{\prime \prime}} \cdot 1$ & $\left.I^{*}\right)$ \\
\hline 25 & 92233 & - & - 13.0 & -I I 44.0 & 2.0 & -184641.0 & 2 \\
\hline 29 & $\begin{array}{lll}8 & 3^{6} & 3^{8}\end{array}$ & + & $\begin{array}{ll}3 & 22.5\end{array}$ & -025.1 & 02145.1 & $\begin{array}{ll}3 & 21.1\end{array}$ & 3 \\
\hline 30 & $\begin{array}{lll}8 & 25 & 0\end{array}$ & - & I 11.6 & $+\quad 56.0$ & $\begin{array}{l}02521.7 \\
\text {. }\end{array}$ & -25 I5 27.2 & 4 \\
\hline Feb. $\quad 1$ & 82136 & + & I 47.9 & - 93.8 & - & - & 5 \\
\hline 4 & $8 \quad 26 \quad 35$ & + & 047.2 & +821.5 & 04145.2 & 4117.8 & 6 \\
\hline
\end{tabular}

with an approximate annual proper motion:

$$
\text { -0.011 }-0.52
$$

\begin{tabular}{|c|c|c|c|}
\hline * & $\alpha$ & $\delta$ & Authority \\
\hline $\mathbf{I}$ & $23^{h} 30^{m} \times 8: 80$ & $-7^{\circ} 45^{\circ} 29^{\circ} \circ$ & Yarnall 10420 \\
\hline 2 & 0615.00 & -18 $34 \quad 57.0$ & Yarnall 54 \\
\hline 3 & 01822.55 & $-2425^{6.0}$ & Yarnall 170 \\
\hline 4 & - $26 \quad 33.34$ & -251723.1 & Cape Cat. 192 \\
\hline 6 & 04058.00 & $\begin{array}{lll}-30 & 49 & 39.3\end{array}$ & Cape Cat. 307 \\
\hline
\end{tabular}

U. S. Naval Observatory Washington 1884 March 6.

E. Frisby.

Observations of Comet Pons-Brooks, made at the Observatory Melbourne.

Adopted places of comparisonstars for abovedates.

Observatory Melbourne 1884 Febr. 8.

") $\Delta \delta$ resp. $\circlearrowright$ app. stimmt nicht mit dem Orte des Vergleichsterns.

Rob. L. F. Ellery. 